\title{
An improved SPE-LC-MS/MS method for multiclass endocrine disrupting compound determination in tropical estuarine sediments
}

\begin{abstract}
Estuary sediments are one of the important components of coastal ecosystems and have been regarded as a sink for various types of organic pollutants. Organic pollutants such as endocrine disrupting compounds (EDCs) which have been associated with various environmental and human health effects were detected in the estuary sediment at trace level. Considering various interferences that may exist in the estuarine sediment, a sensitive and selective method, capable of detecting multiclass EDC pollutants at the trace levels, needs to be developed and optimized to be applied for environmental analysis. A combination of Soxhlet extraction followed by offline solid phase extraction (SPE) cleaned up with detection based on LC triple quadrupole MS was optimized and validated in this study. The targeted compounds consisted of ten multiclass EDCs, namely, diclofenac, primidone, bisphenol A, estrone (E1), 17 $\beta$-estradiol (E2), 17 $\alpha$-ethynylestradiol (EE2), 4-octylphenol (4-OP), 4nonylphenol (4-NP), progesterone, and testosterone. The method showed high extraction efficiency with percentage of recovery from $78 \%$ to $108 \%$ and excellent sensitivity with detection limit between 0.02ngg-1 and 0.81ngg-1. Excellent linearity from 0.991 to 0.999 was achieved for the developed compounds and the relative standard deviation was less than $18 \%$, an indication of good precision analysis. Evaluation of the matrix effects showed ionization suppression for all the developed compounds. Verification of the method was carried out by analyzing the estuarine sediment collected from Langat River. The analyzed estuarine sediments showed a trace concentration of diclofenac, bisphenol A, progesterone, testosterone, primidone, and E1. However, E2, EE2, 4-OP, and 4-NP were below the method's detection limit. Diclofenac exhibited the highest concentration at 2.67ngg-1 followed by bisphenol A (1.78ngg-1) while E1 showed the lowest concentration at 0.07ngg1.
\end{abstract}

Keyword: Endocrine disrupting compounds (EDCs); Estuarine sediment; LC-MS/MS; Matrix effect; Solid phase extraction; Soxhlet 